\title{
CYTOLOGICAL ASPECTS OF ANTIMICROBIAL ANTIBIOSIS
}

\author{
III. Cytologically Distinguishable Stages in Antibiotic Action \\ of Colistin Sulfate on Escherichia coli
}

JEREMY J. KAYE AND GEORGE B. CHAPMAN

Department of Anatomy, Cornell University Medical College, New York, New York

Received for publication 1 May 1963

\begin{abstract}
KAYE, Jeremy J. (Cornell University Medical College, New York, N.Y.) ANd GEorge B. ChaPMAN. Cytological aspects of antimicrobial antibiosis. III. Cytologically distinguishable stages in antibiotic action of colistin sulfate on Escherichia coli. J. Bacteriol. 86:536-543. 1963.Broth cultures of Escherichia coli were subjected to a constant concentration of colistin sulfate for varying periods of time. Controls and treated cells were fixed, dehydrated, and embedded in methacrylate, and ultrathin sections were examined in an electron microscope. Three stages in the antibiotic process were discerned. Stage 1 was characterized by a disruption of the axial orientation of the nuclear material and by an invasion of nuclear areas by tufts of material presumably of cytoplasmic origin; no loss of cellular contents could be detected cytologically. Stage 2 was characterized by the loss of nuclear material and by a loss of typical cytoplasmic granularity, an increase in cytoplasmic electron density, and an agglomeration of the cytoplasm into packed tufts of material; in contrast to the nuclear material, there was no loss of cytoplasmic material in this stage. Stage 3 was characterized by the loss of the altered cytoplasmic material but with the persistence of mesosomes, plasma membrane, and cell wall. Speculation that each and all of these changes might have resulted from an altered intracellular milieu secondary to a primary effect of the antibiotic on the plasma membrane is presented.
\end{abstract}

Earlier studies of the action of antibiotics on the bacterial cell indicated that certain ultrastructural alterations might be associated with such treatment. The treatment of bacteria with chlortetracycline resulted in the formation of a specific annular form of the nucleus (Kellenberger and Ryter, 1955); treatment with penicillin resulted in what has been described as a spadelike alteration of developing cross walls, and a thinning of the cell wall (Murray, Francombe, and Mayall, 1959). In the latter study, the nucleus and cytoplasm unfortunately were not well preserved.

The striking loss of the nuclear material and homogenization of cytoplasmic constituents associated with the exposure of a strain of Escherichia coli to the antibiotic colistin sulfate (Chapman, 1962a), as well as the essentially identical phenomenon observed in a strain of Pseudomonas aeruginosa and the total homogenization of intracellular constituents in a strain of Bacillus megaterium (Chapman, 1962b), suggested the possibility and desirability of experiments designed to elucidate the various cytologically distinguishable stages in the genesis of these observed structural alterations. These previous studies clearly indicated that the response of the bacterial cell to an antibiotic is dependent on the amount of the antibiotic present, and, for a given concentration, that the response is dependent upon the time of exposure. These parameters obviously required further consideration and elucidation. (A report of the nature and action of colistin sulfate has been made by Schwartz et al., 1960.)

This experiment was designed to determine whether a consideration of the parameter of time-action would provide certain clues to the mechanism of nuclear material loss and cytoplasmic homogenization; in addition, such an experiment, it was anticipated, might provide a broader base for the generalization of the response to this antibiotic, since a different strain was to be utilized.

The experiment described here forms a logical part of a continuing attempt to elucidate primarily cytological aspects of the mechanism of action of antibiotics, but also to correlate the observable cytological alterations with the si- 
multaneously occurring metabolic alterations. By such attempts, it is hoped that the everimportant relationship between structure and function may be clarified in the area of antimicrobial antibiosis.

\section{Materials and Methods}

A colistin sulfate-sensitive strain of $E$. coli was used in this experiment. Cultures were grown for $18 \mathrm{hr}$ at $37 \mathrm{C}$ from one loopful of inoculum in $10 \mathrm{ml}$ of a medium consisting of $0.8 \%$ nutrient broth, $1.0 \%$ yeast extract, $1.0 \%$ Tryptone (all obtained from Difco), and $0.8 \% \mathrm{NaCl}$. After the preliminary incubation for $18 \mathrm{hr}$, an equal volume of sterile culture medium was added to all cultures. In the case of the test samples, colistin sulfate $(652.5 \mu \mathrm{g}$ of base $/ \mathrm{mg})$ was included in the added medium so that the final concentration in all the test samples was 1000 $\mu \mathrm{g} / \mathrm{ml}$; no antibiotic was included in the medium added to control samples. Test samples were then returned to the incubator at $37 \mathrm{C}$.

Control samples and samples which were removed from the incubator at $15,30,45,60,75$, 90,120 , and $150 \mathrm{~min}$ were fixed immediately, according to the method of Kellenberger, Ryter, and Séchaud (1958). The fixed samples were washed briefly in the buffer, washed for $2 \mathrm{hr}$ in a buffered $0.5 \%$ uranyl acetate solution, dehydrated by passage through an ethanol series, and embedded in a mixture of ethyl and $n$-butyl methacrylates to which $0.5 \%$ uranyl nitrate had been added, in an attempt to reduce polymerization damage (Ward, 1958), and $1.5 \%$ Luperco CDB (Wallace \& Tiernan, Inc., Buffalo, N.Y.) had been added as catalyst and plasticizer. Polymerization was carried out at $60 \mathrm{C}$ for $18 \mathrm{hr}$.

Ultrathin sections were cut with a Porter-Blum ultramicrotome; the sections were flattened if necessary with toluene vapor (Satir and Peachey, 1958), collected on collodion-coated no. 2213 copper grids (E. F. Fullam, Inc., Schenectady, N.Y.), and stained with uranyl acetate (Watson, 1958). Sections were examined in an RCA EMU2-D electron microscope, and photographic records were taken on medium-contrast plates (Eastman Kodak Co., Rochester, N.Y.).

\section{Results and Discussion}

Figures 1 to 3 represent the appearance of cells from a control culture of the $E$. coli strain. The cell wall is clearly seen, and the plasma membrane usually may be distinguished where the plane of sectioning is favorable. Nuclear areas, low in electron density and having an axial distribution, may be noted, and the granular cytoplasm composed predominantly of ribonucleoprotein particles is clearly seen. Peripheral bodies (Chapman and Hillier, 1953) or mesosomes (FitzJames, 1960) were very rarely seen in control cultures, and then only indistinctly. No appreciable plasmolysis was noted in control cells.

Figures 4 to 8 illustrate the appearance of cells in the first of three cytologically distinguishable stages of the antibiosis after treatment with $1000 \mu \mathrm{g} / \mathrm{ml}$ of colistin sulfate. Note that the cells depicted in Fig. 4 have been exposed to the antibiotic for $30 \mathrm{~min}$, whereas the cells of Figs. 5-8 have been exposed for $15 \mathrm{~min}$. It would be well to recall that a rather large population of cells is being dealt with in each of these samples. Furthermore, the various cells of any such population may be expected to differ in their relative susceptibilities to any antibiotic agent, in their metabolic state, and as regards their stage in the divisional cycle. Thus, it is both reasonable and anticipated that some structural overlap as regards time samples should be found in the arrangement of the antibiosis into the proposed three stages. It remains the task of the cytologist to analyze these populations and the relative distributions of cell types and, by means of this analysis, to resolve the problem in terms of the process which is interrupted by the sampling procedures, and to provide a comprehensive presentation of the process. Analysis of the distribution of cell types in the process of antibiosis by colistin sulfate has shown a remarkable segregation of the cell type described as being in stage 1 in the very earliest of the time samples, of the cell type of stage 2 in the middle time samples, and of the cell type of stage 3 in the very latest samples; the expected overlap was encountered.

Note that there is no evidence of plasmolysis in the cells (Fig. 4 to 8 ). This may be taken as some indication of the absence of severe hyperosmotic stress. This would, in turn, tend to indicate that the observed alterations are not a representation of hyperosmotic phenomena.

The first cytologically distinguishable evidence of alteration of these cells is a disruption of the normal axial distribution of the nuclear material, a disaxialization. This alteration is particularly well demonstrated in Fig. 5 and 6 , two adjacent sections of the same cell, and in Fig. 7, thought to be another section of the same cell. 


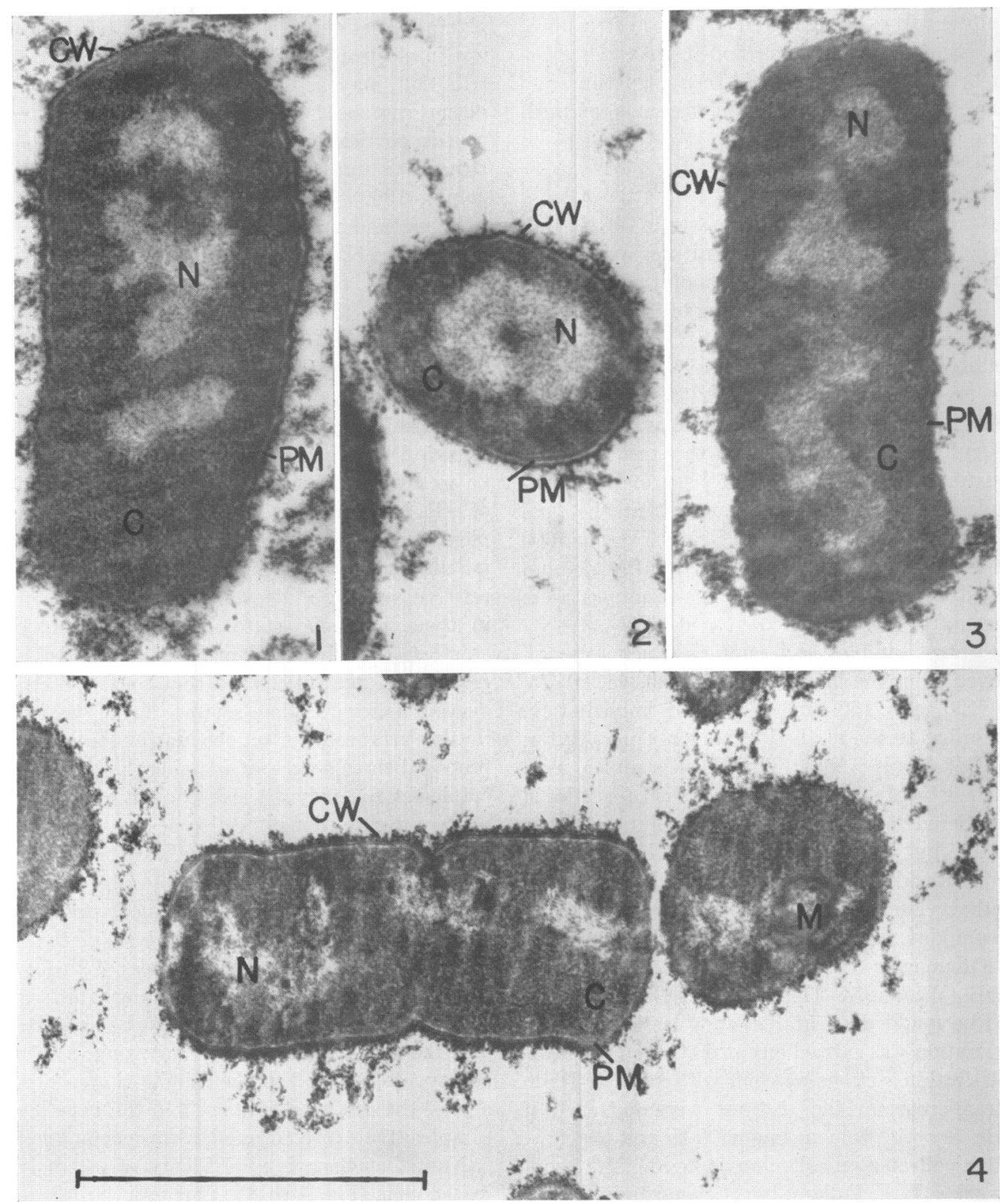

FIG. 1 to 3. Typical cytology of untreated Escherichia coli (control). Cell wall (CW), plasma membrane $(P M)$, granular cytoplasm $(C)$, and low electron density nuclear areas of axial orientation $(N)$ may be seen. Each magnification mark represents $1 \mu$, unless otherwise indicated. The magnification mark on Fig. 4 applies to Fig. 1 to 4.

FIG. 4. Colistin sulfate-treated cells $(1000 \mu \mathrm{g} / \mathrm{ml}, 30 \mathrm{~min})$ in stage 1 . These cells show an early stage in the loss of the axial orientation of the nuclear material. A prominent mesosome $(M)$ presents a profile of densely packed membranous elements. 

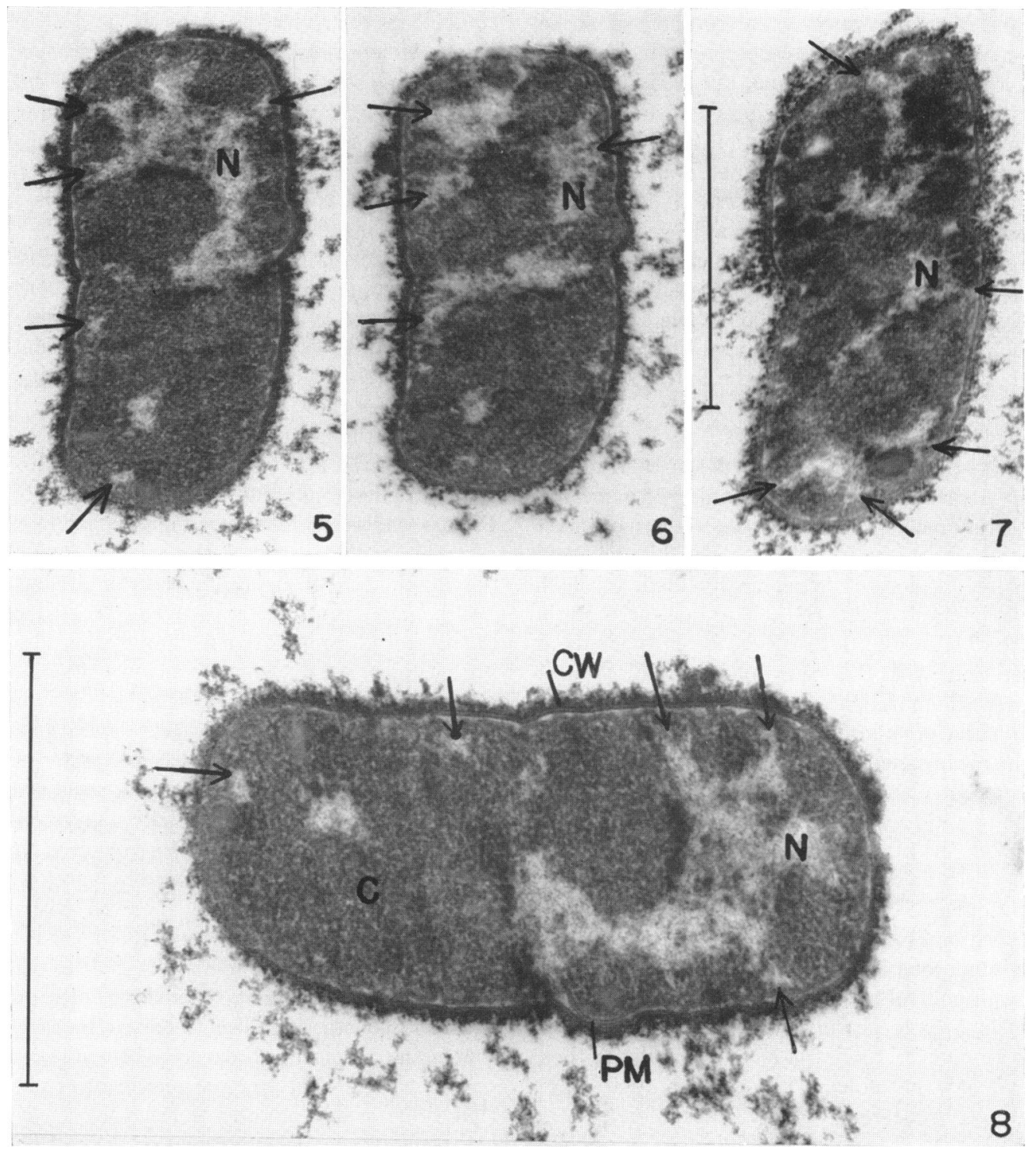

FIG. 5 to 7. Colistin sulfate-treated cells, $(1000 \mu \mathrm{g} / \mathrm{ml}, 15 \mathrm{~min})$ in stage 1 . This cell shows the disaxialization of the nuclear material. Arrows indicate areas of margination of the nuclear material, its apposition to the plasma membrane-cell wall complex. Note that Fig. 5 and 6 are adjacent sections, and Fig. 7 is thought to be another section of the same cell. The magnification mark on Fig. 7 applies to Fig. 5 to 7.

FIG. 8. Higher magnification of cell in Fig. 5 showing margination of nuclear material in areas indicated by arrows.

These same figures also demonstrate a further extension of the disaxialization noted above as a characteristic of the cells of stage 1 ; this is a margination of the nuclear material, i.e., a migration of the deoxyribonucleic acid so that it abuts immediately upon the plasma membrane- cell wall complex. This is particularly well seen at the areas indicated by arrows in Fig. 5 to 8 . This phenomenon is reminiscent of the margination of chromatin material reported to occur a few minutes after infection with bacteriophage (Mudd et al., 1953), although there is no evidence 
to posit a similar functional alteration and mechanism. The present study does not elucidate the etiology of such phenomena, but it is interesting to speculate about the possibility of such changes coming about by an alteration of the selective permeability of the plasma membrane and, consequently, a change in the intracellular milieu of these cells. The alteration of aggregation of the chromatin material as an indication of altered ionic equilibria has been discussed previously (Whitfield and Murray, 1956). The implication of magnesium ion in membrane "stabilization" and its possible relation to the antibiosis by another antibiotic, novobiocin, has appeared in the recent literature (Brock, 1962).

Note that the cytoplasm of the cells in Fig. 4 to 8 shows the typical granularity associated with ribonucleoprotein particles, as was observed in the control cells. There is, at this stage, no evidence of loss of cytologically distinguishable material from these cells. The nuclear material of the cells (Fig. 4 to 8 ) shows no evidence of alteration, with the exception of the disaxialization and margination. Apparently associated with these phenomena, there occurs what appears to be an invasion of nuclear areas by material of a greater electron density; from its electron density and from the irregularity of the boundary between the nuclear material and the cytoplasm, these invading materials are interpreted as tufts of cytoplasm.

Mesosomes appear to become more prominent and numerous in the cells of stage 1 than they were in control cells. One is particularly evident (M) in Fig. 4. The appearance of mesosomes in this strain of $E$. coli is not entirely similar to those previously described (Fitz-James, 1960), presenting profiles of densely packed concentric membranous elements reminiscent of myelin figures; the appearance is somewhat similar to that of mesosomes observed in actinomycetes (Glauert and Hopwood, 1959, 1960).

Stage 1 in the antibiotic action of colistin sulfate is characterized by an early disaxialization and margination of the nuclear material, and by an invasion of nuclear areas by tufts of cytoplasmic material; there is no cytologically distinguishable loss of cellular contents in this stage of the process.

Figures 9 and 10 represent the appearance of cells of stage 2 in the antibiotic action of colistin sulfate on $E$. coli. The characteristic features of this stage are the frank loss of nuclear mate- rial, increase in electron density, loss of characteristic granularity, and homogenization of cytoplasmic constituents. As reported earlier (Chapman, 1962a,b), the loss of nuclear material presumably represents a solubilization of the nuclear material and its subsequent loss. It is not presently known whether this loss is into the culture medium, or whether the loss occurs owing to subsequent extraction in the fixation, dehydration, and embedding procedures; correlative cytological and biochemical studies are presently underway in the hopes of elucidating this matter. In this regard, it is interesting to recall studies showing the release of soluble materials absorbing ultraviolet light at $260 \mathrm{~m} \mu$ when bacteria are treated with polymyxin or cetyltrimethylammonium ion (Newton, 1953). Fluorescent derivatives and fluorescence microscopy have demonstrated the site of action of polymyxin to be the cell membrane (Newton, 1955), and electron microscopy of treated cells in shadowed preparations showed the loss of intracellular constituents (Newton, 1953). The cell membrane has also been implicated by electron microscopy of shadowed preparations of disrupted bacteria which had been treated with polymyxin (Few, 1954), and by shadowed preparations of Leptospira (Bystricky, Ladzianska, and Halaša, 1962). Protoplasts prepared from polymyxin-treated cells showed differences when shadowed preparations were observed with the electron microscope (Newton, 1955). Cetyltrimethylammonium ion, demonstrated to cause the loss of constituents absorbing ultraviolet light at $260 \mathrm{~m} \mu$, has been reported to cause a contraction of intracellular constituents as observed in shadowed preparations (Salton et al., 1951). Tyrocidine, another surface-active agent, has been implicated to cause an alteration in the permeability barrier of bacteria and to cause cellular disruption as observed in shadowed preparations (Mitchell and Crowe, 1947). Thus, there are a number of agents whose primary action can be shown to be at the plasma membrane, and which yet cause the loss of certain cellular constituents, nucleic acids being demonstrated in some cases. These considerations are most interesting in terms of the mode of action of colistin sulfate, especially as regards possible correlations of the functional and structural aspects.

Some finely filamentous material can be noted in the areas occupied by the nuclear material; 


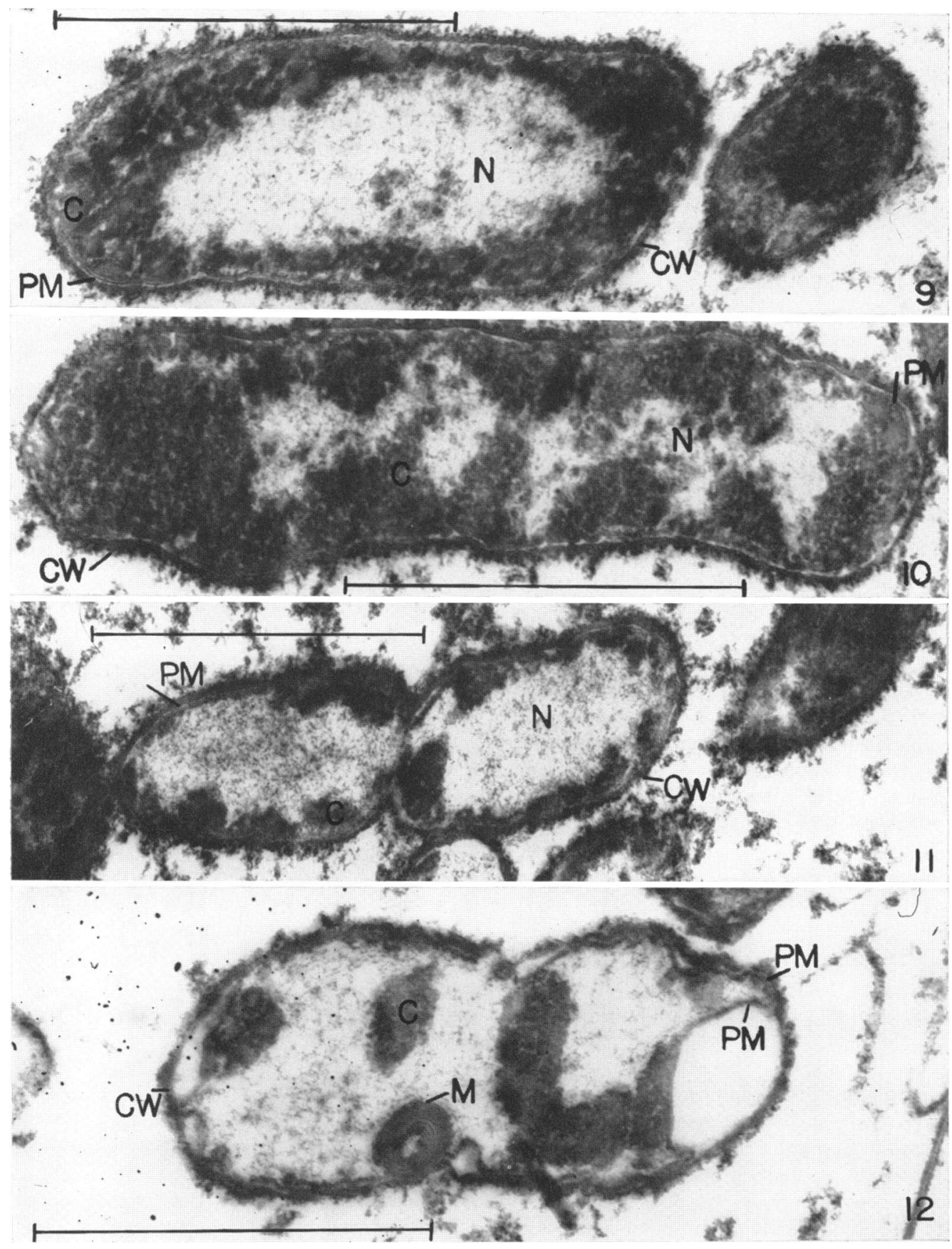

FIG. 9. Colistin sulfate-treated cell $(1000 \mu \mathrm{g} / \mathrm{ml}, 30 \mathrm{~min})$ in stage 2. This cell shows loss of nuclear material, but no loss of cytoplasmic material is detectable. The cytoplasm shows an increase in electron density, homogeneity, and an agglomeration of the cytoplasmic material into closely packed tufts.

FIG. 10. Colistin sulfate-treated cell $(1000 \mu \mathrm{g} / \mathrm{ml}, 30 \mathrm{~min})$ in stage 2. Loss of nuclear material and cytoplasmic changes as in Fig. 9 may be noted.

FIG. 11. Colistin sulfate-treated cell $(1000 \mu \mathrm{g} / \mathrm{ml}, 120 \mathrm{~min})$ in stage 3. A definite loss of cytoplasmic material as well as of nuclear material may be detected.

FIG. 12. Colistin sulfate-treated cell $(1000 \mu \mathrm{g} / \mathrm{ml}, 150 \mathrm{~min})$ in stage 3 . Nuclear and cytoplasmic material loss may be observed, with the persistence of a prominent mesosome (M) presenting a profile of densely packed membranous elements. Remaining cytoplasm appears to be adherent to the plasma membrane. 
similar materials can be noted at the nucleocytoplasmic junction, which might tend to indicate that this material is cytoplasmic and not nuclear in origin. It is, of course, possible that this material has migrated from the nucleus to the cytoplasm.

The cytoplasm (Fig. 9 and 10; typical of stage 2) shows an increase in electron density, with a loss of characteristic granularity and tendency towards homogeneity in electron density, and with an agglomeration or aggregation of the homogeneous material of increased electron density so that the cytoplasmic areas take on the appearance of closely packed tufts of material. Tufts of similarly electron-dense material can be noted to be invading areas formerly occupied by nuclear material. This appears to be similar to the process described for stage 1 , and the fact that these tufts show an increased electron density at this stage gives further evidence of their cytoplasmic origin. The homogenization and increase in electron density of cytoplasmic constituents has been described in earlier papers (Chapman, 1962a,b), although the tuftlike character of the cytoplasm was not observed. The increase in the electron density and homogenization of cytoplasmic constituents and the reaggregation into tufts, with ribonucleoprotein particles particularly being affected, might also be suggested as being due to an alteration of specific membrane permeabilities or transport processes. In this regard, the dependence of the state of aggregation of the ribonucleoprotein particles upon the magnesium ion concentration has already been reported (Tissières and Watson, 1958).

Material is present adherent to the outer lamina of the tripartite wall of $E$. coli, as was the case for stage 1, although the nature of the material remains unknown; mesosomes were observed with relative frequency, although none is depicted in Fig. 9 or 10.

Cells of stage 2 are entirely reminiscent of the appearance of those described earlier (Chapman, $1962 a, b)$ and probably represent the same stage in the process. This is particularly true in Fig. 9, which is an example of the overlap of stages as regards the time period of sampling.

Thus, stage 2 in the antibiotic action of colistin sulfate is characterized by a loss of the nuclear material, a loss of granularity of the cytoplasm, an increase in its electron density, and a reaggregation or agglomeration of the cytoplasm. In contrast to the nuclear material, there is no cytologically distinguishable loss of cytoplasmic constituents in this stage of the process.

Figures 11 and 12 represent the typical appearance of cells in stage 3 of the antibiotic action of colistin sulfate on $E$. coli. The characteristic feature of this stage is the loss of cytoplasmic materials. The small amount of cytoplasmic material which remains is that adjacent, and presumably adherent, to the plasma membrane. The loss of cytoplasmic materials is presumably due, as in the case of the nuclear material, to a solubilization, perhaps secondary to an altered internal ionic environment. Note that the mesosomes remain in this stage; one is particularly apparent in Fig. 12. In fact, these figures provide a prominent feature of the cells observed in stage 3; they are, as before, composed of closely packed membranous elements which when cut in cross section present a myelin-like profile.

In the areas not occupied by residual cytoplasm, the cells of this stage are filled with a very loose particulate and finely filamentous material similar to that noted in the nuclear areas of the cells of stage 2. Again, the origin of this material is thought to be cytoplasmic. Thus, stage 3 is characterized by a loss of cytoplasmic materials and the prominence of mesosomes.

In summary, the exposure of a strain of $E$. coli to a constant concentration of the antibiotic agent colistin sulfate for varying periods of time has resulted in the possibility of separating the antibiotic process into three distinct stages. Stage 1 is characterized by a disaxialization and margination of the nuclear material, and an invasion of nuclear areas by tufts of material which are presumably cytoplasmic in origin. There is no loss of cellular contents in this stage. Stage 2 is characterized by the frank loss of the nuclear material of the treated cells, an increase in the electron density of the cytoplasmic constituents, a loss of typical cytoplasmic granularity and a homogenization of the cytoplasm, and an agglomeration of the latter into a mass of packed tufts of material. There is no distinguishable loss of cytoplasmic constituents in this stage. Stage 3 is characterized by a loss of cytoplasmic constituents with the persistence of cell wall, plasma membrane, mesosomes, and small amounts of adherent cytoplasm. It has been speculated that each and all of these observed ultrastructural alterations might be caused by an altered intracellular ionic milieu, in turn due to a primary 
effect of the antibiotic agent upon the plasma membrane, the site of selective permeability determination.

\section{ACKNOWLEDGMents}

Appreciation is due to the Warner-Lambert Research Institute, especially to Benjamin S. Schwartz and to Robert Smith, for providing the colistin sulfate (Coly-Mycin S) used in these experiments.

This investigation was supported in part by a U.S. Public Health Service Graduate Training Grant $(2 \mathrm{G}-895(\mathrm{C} 2))$ and by research grants E-3517 and 1GS97.

\section{Literature Cited}

Brock, T. D. 1962. Effects of magnesium ion deficiency on Escherichia coli and possible relation to the mode of action of novobiocin. J. Bacteriol. 84:679-682.

Bystrický, V., K. Ladzianska, and M. Halaša. 1962. Electron microscopy of the action of polymyxin on leptospirae. J. Bacteriol. $84: 864-865$.

Chapman, G. B. 1962a. Cytological aspects of antimicrobial antibiosis. I. Cytological changes associated with the exposure of Escherichia coli to colistin sulfate. J. Bacteriol. 84:169-179.

Chapman, G. B. 1962a. Cytological aspects of antimicrobial antibiosis. II. Cytological changes associated with the exposure of Pseudomonas aeruginosa and Bacillus megaterium to colistin sulfate. J. Bacteriol. 84:180185.

Chapman, G. B., and J. Hillier. 1953. Electron microscopy of ultra-thin sections of bacteria. I. Cellular division in Bacillus cereus. J. Bacteriol. 66:362-373.

FEw, A. V. 1954. Electron microscopy of disrupted bacteria treated with Polymyxin E. J. Gen. Microbiol. 10:304-308.

Fitz-James, P. C. 1960. Participation of the cytoplasmic membrane in the growth and spore formation of bacilli. J. Biophys. Biochem. Cytol. 8:507-528.

Glauert, A. M., AND D. A. Hopwood. 1959. A membranous component of the cytoplasm in Streptomyces coelicolor. J. Biophys. Biochem. Cytol. 6:515-516.

Glauert, A. M., And D. A. Hopwood. 1960. The fine structure of Streptomyces coelicolor. I. The cytoplasmic membrane system. J. Biophys. Biochem. Cytol. 7:479-488.
Kellenberger, E., and A. Ryter. 1955. Contribution a l'étude du noyau bacterien. Schweiz. Z. Allgem. Pathol. Bakteriol. 18: 1122-1137.

Kellenberger, E., A. Ryter, and J. Sechaud. 1958. Electron microscope study of DNAcontaining plasms. II. Vegetative and mature phage DNA as compared with normal bacterial nucleoids in different physiological states. J. Biophys. Biochem. Cytol. 4:671-678.

Mitchell, P. D., And G. R. Crowe. 1947. A note on electron micrographs of normal and tyrocidin-lysed Streptococci. J. Gen. Microbiol. $1: 85$.

Mudd, S., J. Hillier, E. H. Beutner, and P. E. Hartman. 1953. Light and electron microscopic studies of Escherichia coli-coliphage interactions. II. The electron microscopic cytology of the $E$. coli $\mathrm{B}-\mathrm{T}_{2}$ system. Biochim. Biophys. Acta 10:153-179.

Murray, R. G. E., W. H. Francombe, and B. H. MAYALL. 1959. The effect of penicillin on the structure of staphylococcal cell walls. Can. J. Microbiol. 5:641-648.

Newton, B. A. 1953. The release of soluble constituents from washed cells of Pseudomonas aeruginosa by the action of polymyxin. J. Gen. Microbiol. 9:54-64.

Newton, B. A. 1955. A fluorescent derivative of polymyxin: its preparation and use in studying the site of action of the antibiotic. J. Gen. Microbiol. $12: 226-236$.

Salton, M. R. J., R. W. Horne, and V. E. CossLETT. 1951. Electron microscopy of bacteria treated with cetyltrimethylammonium bromide. J. Gen. Microbiol. 5:405-407.

Satir, P. G., and L. D. Peachey. 1958. Thin sections. II. A simple method for reducing compression artifacts. J. Biophys. Biochem. Cytol. 4:345-348.

Schwartz, B. S., M. R. Warren, F. A. Barkley, AND L. LANDis. 1960. Microbiological and pharmacological studies of colistin sulfate and sodium colistin methanesulfonate. Antibiot. Ann. 1959-1960, p. 41-60.

Tissières, A., AND J. D. Watson. 1958. Ribonucleoprotein particles from Escherichia coli. Nature 182:778-780.

WARD, R. T. 1958. Prevention of polymerization damage in methacrylate embedding media. J. Histochem. Cytochem. 6:398.

Watson, M. L. 1958. Staining of tissue sections for electron microscopy with heavy metals. J. Biophys. Biochem. Cytol. 4:475-478.

Whitfield, J. F., and R. G. E. Murray. 1956. The effects of the ionic environment on the chromatin structure of bacteria. Can. J. Microbiol. 2:245-260. 